\title{
AN EXPRESSION FOR SOLUTIONS TO THREE-DIMENSIONAL ELASTICITY PROBLEMS IN CYLINDRICAL AND SPHERICAL COORDINATES
}

\author{
By Isamu A. OKUMURA* and Takayoshi ONAKA**
}

\begin{abstract}
Solutions to three-dimensional elasticity problems in cylindrical and spherical coordinates are proposed as useful solutions to non-axially symmetric deformations. The solutions with the aid of potential functions of displacement take into account effects of heat and body forces in general forms. A harmonic vector of potential functions based on Boussinseq's solution is replaced by a vector field consisted of harmonic functions according to cylindrical and spherical coordinates. This method facilitates the determination of potential functions enough for the theory of elasticity. Expressions for potential functions and components of displacement of non-axially symmetric elasticity problems in cylindrical and spherical coordinates are definitely presented in due consideration of practical applications to stress analyses.

Keyword: elasticity, three-dimensional problem, hollow cylinder, hollow sphere.
\end{abstract}

\section{INTRODUCTION}

A number of studies on three-dimensional elasticity problems, even if they are restricted within finite elastic solids such as rectangular prisms, thick plates, circular hollow cylinders and hollow spheres, have been done by many investigators in the course of the long history of the theory of elasticity. The solutions used in their studies are divided broadly into Boussinesq's solution ${ }^{1)}$ governed by linear second-order partial differential equations and Galerkin's solution ${ }^{2)}$ governed by linear fourth-order partial differential equations. These two solutions are classical and fundamental solutions to three-dimensional elasticity problems and are applicable to elasticity problems in arbitrary coordinate systems included in orthogonal curvilinear coordinates. However, after that, more convenient solutions for practical applications according to boundary conditions or coordinate systems have been reported by some investigators.

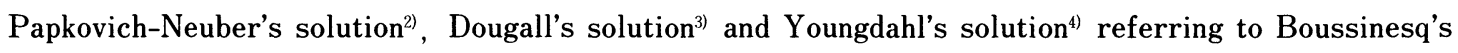
solution have been found. Also, Michell's solution ${ }^{5)}$, Hasegawa's solution ${ }^{6)}$ to axi-symmetric problems and Muki's solution ${ }^{7)}$ to semi-infinite solids referring to Galerkin's solution have been found.

Papkovich-Neuber's solution among these solutions has a defect that two or three components of the vector potential of displacement couple with each other in cylindrical or spherical coordinates. This defect yields a difficult subject with not only the complexity of solving differential equations but also the need for the investigation on an independent relation between each solution. Boussinesq's solution and Galerkin's solution have also this defect in orthogonal curvilinear coordinates except rectangular Cartesian coordinates. This defect has not been settled also in the extended Neuber solution ${ }^{8.99}$ used so far by one of the present authors, though the investigation on an independent relation between each solution has been

* Member of JSCE, Dr. Eng., Professor, Department of Civil Engineering, Kitami Institute of Technology (Koen-cho, Kitami).

** Member of JSCE, Engineer, Department of Civil Engineering, Kitami Institute of Technology. 
done. Youngdahl's solution in cylindrical coordinates has a complexity that Poisson's equation must be solved to determine potential functions of displacement and, after that, appropriate solutions to elasticity problems must be chosen. Though the method of analysis for axi-symmetric problems in spherical coordinates is almost established at the present time ${ }^{10)}$, the studies on non-axially symmetric problems, by the general method making use of potential functions of displacement seem to be a few except for the study ${ }^{11)}$ by one of the present authors. This study using Boussinesq's solution in which potential functions of displacement have been obtained from solving a system of linear partial differential equations with three unknowns and, after that, the investigation on an independent relation between each solution has been accomplished has stated only the result without the process of derivation of the solutions.

As stated above, even at the present time, simple and plain solutions to three-dimensional elasticity problems in orthogonal curvilinear coordinates, for instance, cylindrical or spherical coordinates seem to be not clarified. From this point of view, this paper proposes simple and plain solutions to be available for non-axially symmetric problems in cylindrical and spherical coordinates and indicates concrete expressions for potential functions of displacement and for components of displacement. The solutions proposed in this paper are generalized to taking into account heat and body forces in both cylindrical and spherical coordinates. In case of the absence of body forces, a simplification that all potential functions of displacement as well as them in rectangular Cartesian coordinates reduce to harmonic functions is attempted. This simplification is accomplished by the investigation on an independent relation between each solution and on decomposing the coupled governing equations of a harmonic vector ${ }^{12)}$.

\section{GENERALIZATION OF BOUSSINESQ'S SOLUTION IN ORTHOGONAL CURVI- LINEAR COORDINATES}

If we let $\boldsymbol{u}$ and $\boldsymbol{b}$ a displacement field and a body force field, respectively, the displacement equation of equilibrium for an elastic solid with a temperature field $T$ are as follows:

$$
\nabla^{2} u+\frac{1}{1-2 \nu} \operatorname{grad} \operatorname{div} u-\frac{2 \alpha(1+\nu)}{1-2 \nu} \operatorname{grad} T+\frac{b}{G}=0
$$

in which $G, \nu$ and $\alpha$ denote the shear modulus, Poisson's ratio and the coefficient of linear thermal expansion, respectively. By Helmholtz's theorem ${ }^{13)}$, the body force $b$ can be expressed as :

$\boldsymbol{b}=\operatorname{grad} \beta+\operatorname{rot} \tau$

in which $\beta$ and $\tau$ denote a scalar potential and a vector potential to be determined from given body foces, respectively. Substituting Eq. (2) into Eq. (1), we have

$$
\nabla^{2} u+\frac{1}{1-2 \nu} \operatorname{grad} \operatorname{div} u-\frac{2 \alpha(1+\nu)}{1-2 \nu} \operatorname{grad} T+\frac{1}{G}(\operatorname{grad} \beta+\operatorname{rot} \tau)=0
$$

Though there are some solutions to Eq. ( 3 ), we employ here the following solution based on Boussinesq's solution :

$$
2 G \boldsymbol{u}=\operatorname{grad}\left(\phi_{0}+\boldsymbol{r} \cdot \lambda\right)-4(1-\nu) \lambda+2 \operatorname{rot} \vartheta+\operatorname{grad} \chi
$$

in which $\boldsymbol{r}$ denotes the position vector in orthogonal curvilinear coordinates, and $\phi_{0}, \lambda, \vartheta$ and $\chi$ are a scalar potential of displacement, vector potentials of displacement and a thermoelastic potential of displacement, respectively. Substituting Eq. (4) into Eq. (3) multiplied by $2 G$, we have

$$
\begin{aligned}
& \operatorname{grad}\left[2\left(\frac{1-\nu}{1-2 \nu} \nabla^{2} \phi_{0}+\beta\right)+\frac{2(1-\nu)}{1-2 \nu} r \cdot \nabla^{2} \lambda+\frac{2}{1-2 \nu}\left\{(1-\nu) \nabla^{2} \chi-\alpha E T\right\}\right] \\
& -4(1-\nu) \nabla^{2} \lambda+2 \operatorname{rot}\left(\nabla^{2} \vartheta+\tau\right)=0 \text {. }
\end{aligned}
$$

in which the following relations are used :

$\nabla^{2} \operatorname{grad} \phi_{0}=\operatorname{grad} \nabla^{2} \phi_{0}, \quad \nabla^{2} \operatorname{rot} \vartheta=\operatorname{rot} \nabla^{2} \vartheta, \quad \nabla^{2}(\boldsymbol{r} \cdot \lambda)=r \cdot \nabla^{2} \lambda+2 \operatorname{div} \lambda$

If we have

$$
\nabla^{2} \phi_{0}=-\frac{1-2 \nu}{1-\nu} \beta, \quad \nabla^{2} \chi=\frac{\alpha E}{1-\nu} T, \quad \nabla^{2} \lambda=0, \quad \nabla^{2} \vartheta=-\tau
$$


equation $(5)$ is satisfied for $\nu \neq 1 / 2$. In the case where $\boldsymbol{b}=\mathbf{0}$, we can put that

$$
\beta=0, \tau=\mathbf{0}
$$

From the above equations, equations (7.a,d) become

$$
\nabla^{2} \phi_{0}=0, \quad \nabla^{2} \vartheta=0
$$

Then, equation (4) except $\chi$ yields Boussinesq's solution.

Thus, Boussinesq's solution has been generalized to taking into account heat and body forces.

\section{SOLUTIONS TO THREE-DIMENSIONAL ELASTICITY PROBLEMS IN CYLINDRI- CAL COORDINATES}

If we consider Eq. (4) in cylindrical coordinates $(r, \theta, z)$ as shown in Fig. 1, we have

$$
2 G \boldsymbol{u}=\operatorname{grad}\left(\phi_{0}+\boldsymbol{r} \cdot \lambda\right)-4(1-\nu) \lambda+2 \operatorname{rot} \vartheta+\operatorname{grad} \chi
$$

in which

$$
\begin{aligned}
& \boldsymbol{u}=\left[u_{r}, u_{\theta}, u_{z}\right], \quad \boldsymbol{r}=[r, 0, z], \quad \lambda=\left[\lambda_{r}, \lambda_{\theta}, \lambda_{z}\right], \quad \vartheta=\left[\vartheta_{r}, \vartheta_{\theta}, \vartheta_{z}\right] \\
& \nabla^{2} \phi_{0}=-\frac{1-2 \nu}{1-\nu} \beta, \quad \nabla^{2} \chi=\frac{\alpha E}{1-\nu} T, \quad \nabla^{2} \lambda=0, \quad \nabla^{2} \vartheta=-\tau \\
& \nabla^{2} \equiv \frac{\partial^{2}}{\partial r^{2}}+\frac{1}{r} \frac{\partial}{\partial r}+\frac{1}{r^{2}} \frac{\partial^{2}}{\partial \theta^{2}}+\frac{\partial^{2}}{\partial z^{2}}
\end{aligned}
$$

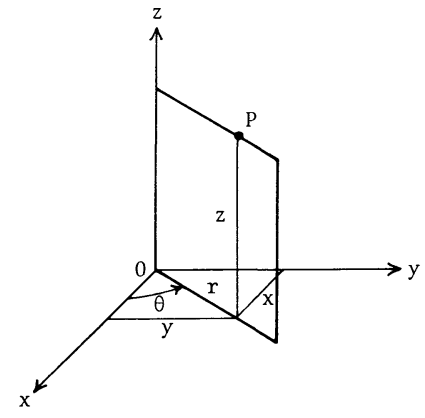

Fig. 1 Cylindrical coordinates

Laplace's equation (12.c) in the vector field can be expressed in the components of vector potential $\lambda$ as

$$
\nabla^{2} \lambda_{r}-\frac{\lambda_{r}}{r^{2}}-\frac{2}{r^{2}} \frac{\partial \lambda_{\theta}}{\partial \theta}=0, \quad \nabla^{2} \lambda_{\theta}-\frac{\lambda_{\theta}}{r^{2}}+\frac{2}{r^{2}} \frac{\partial \lambda_{r}}{\partial \theta}=0, \quad \nabla^{2} \lambda_{z}=0
$$

From the above equations, we find a system of linear partial differential equations with two unknowns, that is, $\lambda_{r}$ and $\lambda_{\theta}$. Though the system can be solved by an ingenious method, the solutions must be checked by independence from the solutions in Eq. (12.d). To avoid this complexity, we replace vector potential $\lambda$ with a certain vector field as

$$
\lambda=\operatorname{grad} \phi_{1}+\frac{\partial}{\partial z}\left(\phi_{3}-\phi_{1}\right) \boldsymbol{e}_{z}=\left[\frac{\partial \phi_{1}}{\partial r}, \frac{1}{r} \frac{\partial \phi_{1}}{\partial \theta}, \frac{\partial \phi_{3}}{\partial z}\right]
$$

in which $\boldsymbol{e}_{z}$ denotes the unit vector, and $\phi_{1}$ and $\phi_{3}$ are harmonic functions satisfying Laplace's equation as

$$
\nabla^{2} \phi_{1}=0, \quad \nabla^{2} \phi_{3}=0
$$

Relation (15) is exactly held to satisfy condition (12. c) that $\lambda$ must be the harmonic vector, as indicated in the following equation :

$$
\nabla^{2} \lambda=\nabla^{2} \operatorname{grad} \phi_{1}+\nabla^{2}\left\{\frac{\partial}{\partial z}\left(\phi_{3}-\phi_{1}\right)\right\} \boldsymbol{e}_{z}=\operatorname{grad} \nabla^{2} \phi_{1}+\frac{\partial}{\partial z}\left(\nabla^{2} \phi_{3}-\nabla^{2} \phi_{1}\right) \boldsymbol{e}_{z}=\mathbf{0}
$$

Substituting Eq. (15) into Eq. (10), we have

$$
2 G \boldsymbol{u}=\operatorname{grad}\left(\phi_{0}+r \frac{\partial \phi_{1}}{\partial r}+z \frac{\partial \phi_{3}}{\partial z}\right)-4(1-\nu)\left[\frac{\partial \phi_{1}}{\partial r}, \frac{1}{r} \frac{\partial \phi_{1}}{\partial \theta}, \frac{\partial \phi_{3}}{\partial z}\right]+2 \operatorname{rot} \vartheta+\operatorname{grad} \chi
$$

The components of displacement, from the above equation, are expressed as :

$$
\begin{aligned}
& 2 G u_{r}=\frac{\partial}{\partial r}\left\{\phi_{0}+r \frac{\partial \phi_{1}}{\partial r}+z \frac{\partial \phi_{3}}{\partial z}-4(1-\nu) \phi_{1}\right\}+2\left(\frac{1}{r} \frac{\partial \vartheta_{z}}{\partial \theta}-\frac{\partial \vartheta_{\theta}}{\partial z}\right)+\frac{\partial \chi}{\partial r} \\
& 2 G u_{\theta}=\frac{1}{r} \frac{\partial}{\partial \theta}\left\{\phi_{0}+r \frac{\partial \phi_{1}}{\partial r}+z \frac{\partial \phi_{3}}{\partial z}-4(1-\nu) \phi_{1}\right\}+2\left(\frac{\partial \vartheta_{r}}{\partial z}-\frac{\partial \vartheta_{z}}{\partial r}\right)+\frac{1}{r} \frac{\partial \chi}{\partial \theta} \text {. } \\
& 2 G u_{z}=\frac{\partial}{\partial z}\left\{\phi_{0}+r \frac{\partial \phi_{1}}{\partial r}+z \frac{\partial \phi_{3}}{\partial z}-4(1-\nu) \phi_{3}\right\}+2\left\{\frac{1}{r} \frac{\partial\left(r \vartheta_{\theta}\right)}{\partial r}-\frac{1}{r} \frac{\partial \vartheta_{r}}{\partial \theta}\right\}+\frac{\partial \chi}{\partial z}
\end{aligned}
$$

in which the governing equations of $\phi_{0}, \chi, \vartheta, \phi_{1}$ and $\phi_{3}$ are expressed in Eqs. (12. a, b, d) and (16. a, b), respectively. In the case where $\boldsymbol{b}=\mathbf{0}$, on considering Eqs. (8. a, b) and independence from the potential 
functions of displacement in Eq. (15), we can put that

$\vartheta_{r}=0, \vartheta_{\theta}=0$

Then, $\phi_{0}$ and $\vartheta_{z}$ reduce to harmonic functions as

$\nabla^{2} \phi_{0}=0, \quad \nabla^{2} \vartheta_{z}=0$

The components of displacement in case of zero body forces and ze ro temperature are obtained from putting $\chi=0, \vartheta_{r}=0$ and $\vartheta_{\theta}=0$ in Eqs. $(19 . a \sim c)$ as

$$
\begin{aligned}
& 2 G u_{r}=\frac{\partial}{\partial r}\left\{\phi_{0}+r \frac{\partial \phi_{1}}{\partial r}+z \frac{\partial \phi_{3}}{\partial z}-4(1-\nu) \phi_{1}\right\}+\frac{2}{r} \frac{\partial \vartheta_{z}}{\partial \theta} \\
& 2 G u_{\theta}=\frac{1}{r} \frac{\partial}{\partial \theta}\left\{\phi_{0}+r \frac{\partial \phi_{1}}{\partial r}+z \frac{\partial \phi_{3}}{\partial z}-4(1-\nu) \phi_{1}\right\}-2 \frac{\partial \vartheta_{z}}{\partial r} \\
& 2 G u_{z}=\frac{\partial}{\partial z}\left\{\phi_{0}+r \frac{\partial \phi_{1}}{\partial r}+z \frac{\partial \phi_{3}}{\partial z}-4(1-\nu) \phi_{3}\right\}
\end{aligned}
$$

in which the governing equations of $\phi_{1}, \phi_{3}, \phi_{0}$ and $\vartheta_{z}$ are expressed in Eqs. (16. a, b) and (21. a, b), respectively. Hence, it is found that all potential functions of displacement reduce to the harmonic functions. This result yields simple and plain solutions in which the potential functions of displacement are easily determined and no investigation on an independent relation between each solution is needed. The harmonic functions in Eqs. (16. a, b) and (21. a, b) in the cylindrical coordinates are easily obtained by the method of separation of variables. The potential functions of displacement for non-axially symmetric problems of circular hollow cylinders are as follows :

$$
\begin{aligned}
& \phi_{0}=\sum_{m=0}^{\infty} \sum_{s=1}^{\infty} \cos m \theta\left\{J_{m}\left(\alpha_{m s} r\right)\left(A_{m s}^{(1)} \cosh \alpha_{m s} z+L_{m s}^{(1)} \sinh \alpha_{m s} z\right)+Y_{m}\left(\alpha_{m s} r\right)\left(A_{m s}^{(2)} \cosh \alpha_{m s} z\right.\right. \\
& \left.\left.+L_{m s}^{(2)} \sinh \alpha_{m s} z\right)\right\}+\sum_{m=0}^{\infty} \sum_{n=1}^{\infty} \cos m \theta \cos \beta_{n} z\left\{D_{m n}^{(1)} I_{m}\left(\beta_{n} r\right)+D_{m n}^{(2)} K_{m}\left(\beta_{n} r\right)\right\} \\
& \phi_{1}=\sum_{m=0}^{\infty} \sum_{n=1}^{\infty} \cos m \theta \cos \beta_{n} z\left\{F_{m n}^{(1)} I_{m}\left(\beta_{n} r\right)+F_{m n}^{(2)} K_{m}\left(\beta_{n} r\right)\right\} \\
& \phi_{3}=\sum_{m=0}^{\infty} \sum_{s=1}^{\infty} \cos m \theta\left\{J_{m}\left(\alpha_{m s} r\right)\left(C_{m s}^{(1)} \cosh \alpha_{m s} z+M_{m s}^{(1)} \sinh \alpha_{m s} z\right)+Y_{m}\left(\alpha_{m s} r\right)\left(C_{m s}^{(2)} \cosh \alpha_{m s} z\right.\right. \\
& \left.\left.+M_{m s}^{(2)} \sinh \alpha_{m s} z\right)\right\} \\
& \vartheta_{z}=\sum_{m=0}^{\infty} \sum_{s=1}^{\infty} \sin m \theta\left\{J_{m}\left(\alpha_{m s} r\right)\left(B_{m s}^{(1)} \cosh \alpha_{m s} z+G_{m s}^{(1)} \sinh \alpha_{m s} z\right)+Y_{m}\left(\alpha_{m s} r\right)\left(B_{m s}^{(2)} \cosh \alpha_{m s} z\right.\right. \\
& \left.\left.+G_{m s}^{(2)} \sinh \alpha_{m s} z\right)\right\}+\sum_{m=0}^{\infty} \sum_{n=1}^{\infty} \sin m \theta \cos \beta_{n} z\left\{E_{m n}^{(1)} I_{m}\left(\beta_{n} r\right)+E_{m n}^{(2)} K_{m}\left(\beta_{n} r\right)\right\}
\end{aligned}
$$

in which $J_{m}\left(\alpha_{m s} r\right)$ and $Y_{m}\left(\alpha_{m s} r\right)$ are Bessel functions of the first and of the second kind, respectively. Also, $I_{m}\left(\beta_{n} r\right)$ and $K_{m}\left(\beta_{n} r\right)$ are the modified Bessel functions of the first and of the second kind, respectively. Furthermore, $\alpha_{m s}$ and $\beta_{n}$ are characteristic values to be chosen according to given boundary conditions, and $A_{m s}^{(1)}$ to $E_{m n}^{(2)}$ are arbitrary constants to be determined from given boundary conditions. It is convenient to replace circular function $\cos \beta_{n} z$ in Eqs. (23. a, b, d) with $\sin \beta_{n} z$ in such a case as a cantilever cylinder subjected to a edge load. If we affix superscripts (1) and (2) to components of displacement derived from Bessel functions and the modified Bessel functions, respectively, the components of displacement with superscript (1) are as follows:

$$
\begin{aligned}
& 2 G u_{r}^{(1)}=\sum_{m=0}^{\infty} \sum_{s=1}^{\infty} \frac{\alpha_{m s}}{2} \cos m \theta\left\{J_{m-1}\left(\alpha_{m s} r\right) f_{1}(z)-J_{m+1}\left(\alpha_{m s} r\right) f_{2}(z)+Y_{m-1}\left(\alpha_{m s} r\right) g_{1}(z)\right. \\
& \left.-Y_{m+1}\left(\alpha_{m s} r\right) g_{2}(z)\right\} \\
& 2 G u_{\theta}^{(1)}=\sum_{m=0}^{\infty} \sum_{s=1}^{\infty}\left(-\frac{\alpha_{m s}}{2}\right) \sin m \theta\left\{J_{m-1}\left(\alpha_{m s} r\right) f_{1}(z)+J_{m+1}\left(\alpha_{m s} r\right) f_{2}(z)+Y_{m-1}\left(\alpha_{m s} r\right) g_{1}(z)\right. \\
& \left.+Y_{m+1}\left(\alpha_{m s} r\right) g_{2}(z)\right\} \\
& 2 G u_{z}^{(1)}=\sum_{m=0}^{\infty} \sum_{s=1}^{\infty} \alpha_{m s} \cos m \theta\left\{J_{m}\left(\alpha_{m s} r\right) f_{3}(z)+Y_{m}\left(\alpha_{m s} r\right) g_{3}(z)\right\}
\end{aligned}
$$


in which

$$
\begin{aligned}
& f_{1}(z)=\left(A_{m s}^{(1)}+2 B_{m s}^{(1)}\right) \cosh \alpha_{m s} z+\left(L_{m s}^{(1)}+2 G_{m s}^{(1)}\right) \sinh \alpha_{m s} z+C_{m s}^{(1)} \alpha_{m s} z \sinh \alpha_{m s} z \\
& +M_{m s}^{(1)} \alpha_{m s} z \cosh \alpha_{m s} z \\
& f_{2}(z)=\left(A_{m s}^{(1)}-2 B_{m s}^{(1)}\right) \cosh \alpha_{m s} z+\left(L_{m s}^{(1)}-2 G_{m s}^{(1)}\right) \sinh \alpha_{m s} z+C_{m s}^{(1)} \alpha_{m s} z \sinh \alpha_{m s} z \\
& +M_{m s}^{(1)} \alpha_{m s} z \cosh \alpha_{m s} z \\
& +C_{m s}^{(1)} \alpha_{m s} z \cosh \alpha_{m s} z+M_{m s}^{(1)} \alpha_{m s} z \sinh \alpha_{m s} z
\end{aligned}
$$

$2 G u_{\theta}^{(2)}=\sum_{m=0}^{\infty} \sum_{n=1}^{\infty}\left(-\frac{\beta_{n}}{2}\right) \sin m \theta \cos \beta_{n} z\left[I_{m-1}\left(\beta_{n} r\right)\left\{D_{m n}^{(1)}+(m-4+4 \nu) F_{m n}^{(1)}+2 E_{m n}^{(1)}\right\}\right.$

$$
\left.-I_{m+1}\left(\beta_{n} r\right)\left\{D_{m n}^{(1)}-(m+4-4 \nu) F_{m n}^{(1)}-2 E_{m n}^{(1)}\right]\right]+\sum_{m=0}^{\infty} \sum_{n=1}^{\infty} \frac{\beta_{n}}{2} \sin m \theta \cos \beta_{n} z
$$$$
\cdot\left[K_{m-1}\left(\beta_{n} r\right)\left\{D_{m n}^{(2)}+(m-4+4 \nu) F_{m n}^{(2)}+2 E_{m n}^{(2)}\right\}-K_{m+1}\left(\beta_{n} r\right)\left\{D_{m n}^{(2)}\right.\right.
$$$$
\left.-(m+4-4 \nu) F_{m n}^{(2)}-2 E_{m n}^{(2)}\right]
$$

$$
\begin{aligned}
2 G u_{z}^{(2)}= & \sum_{m=0}^{\infty} \sum_{n=1}^{\infty}\left(-\beta_{n}\right) \cos m \theta \sin \beta_{n} z\left\{I_{m}\left(\beta_{n} r\right)\left(D_{m n}^{(1)}+m F_{m n}^{(1)}\right)+F_{m n}^{(1)}\left(\beta_{n} r\right) I_{m+1}\left(\beta_{n} r\right)\right\} \\
& +\sum_{m=0}^{\infty} \sum_{n=1}^{\infty}\left(-\beta_{n}\right) \cos m \theta \sin \beta_{n} z\left\{K_{m}\left(\beta_{n} r\right)\left(D_{m n}^{(2)}+m F_{m n}^{(2)}\right)-F_{m n}^{(2)}\left(\beta_{n} r\right) K_{m+1}\left(\beta_{n} r\right)\right\}
\end{aligned}
$$

The required components of displacement are the sum of two amounts with superscripts (1) and (2). If we have components of strain in the cylindrical coordinates from the components of displacement in Eqs. (24. a) to (26. c), components of stress can be obtained from Hooke's law, though they are omitted here.

Thus, the generalized solution taking into account heat and body forces has been proposed as a solution to three-dimensional elasticity problems in the cylindrical coordinates. Also, the potential functions of displacement and the components of displacement for non-axially symmetric problems in the absence of a temperature and of body forces have been indicated as a particular case of its solution.

\section{ADDITIONAL SOLUTIONS TO NON-AXIALLY SYMMETRIC PROBLEMS IN CYLIN- DRICAL COORDINATES}

The solutions stated in the latter half of Chap. 3 are fundamental solutions to non-axially symmetric problems. However, there are some cases in which given boundary conditions are not satisfied by these fundamental solutions alone. Hence, an additional solution as hereafter provided for is needed. However, a certain solution, even if it is taken as an additional solution, is not always an appropriate solution. The desirable solution as an additional solution is one, as much as possible, without coupling with the fundamental solutions when boundary conditions are prescribed. From this point of view, the solution to the state of plane strain seems to be the most appropriate solution as an additional solution. If we consider the case of zero body forces and zero temperature, the potential functions of displacement in Eqs. (16. a) and (21.a, b) are expressed in the following harmonic functions:

$$
\phi_{0,0}=\sum_{m=2}^{\infty} D_{m 0}^{(1)} \cos m \theta r^{m}+\sum_{m=1}^{\infty} D_{m 0}^{(2)} \cos m \theta r^{-m}+\sum_{m=1}^{\infty} \bar{D}_{m 0}^{(1)} \cos m \theta\left\{\frac{r^{m+2}}{2}-(m+1) r^{m} z^{2}\right\}
$$




$$
\begin{aligned}
& +\sum_{m=2}^{\infty} \bar{D}_{m 0}^{(2)} \cos m \theta\left\{\frac{r^{-m+2}}{2}+(m-1) r^{-m} z^{2}\right\} \\
& \phi_{1,0}=\sum_{m=1}^{\infty} F_{m 0}^{(1)} \cos m \theta\left\{\frac{r^{m+2}}{2}-(m+1) r^{m} z^{2}\right\}+\sum_{m=2}^{\infty} F_{m 0}^{(2)} \cos m \theta\left\{\frac{r^{-m+2}}{2}+(m-1) r^{-m} z^{2}\right\} \\
& \vartheta_{z, 0}=\sum_{m=1}^{\infty} E_{m 0}^{(1)} \sin m \theta\left\{\frac{r^{m+2}}{2}-(m+1) r^{m} z^{2}\right\}+\sum_{m=2}^{\infty} E_{m 0}^{(2)} \sin m \theta\left\{\frac{r^{-m+2}}{2}+(m-1) r^{-m} z^{2}\right\}
\end{aligned}
$$

in which $D_{m 0}^{(1)}$ to $E_{m 0}^{(2)}$ are arbitrary constants. Substituting the potential functions of displacement in Eqs. $(27 . a \sim c)$ into Eqs. $(22 . a \sim c)$ and putting

$$
2 G u_{z}^{(0)}=0
$$

we obtain the following relations:

$$
\bar{D}_{m 0}^{(1)}=-m F_{m 0}^{(1)}, \bar{D}_{m 0}^{(2)}=m F_{m 0}^{(2)}
$$

Furthermore, eliminating the term of $z^{2}$ included in two components of displacement, that is, $2 G u_{r}^{(0)}$ and $2 G u_{\theta}^{(0)}$, we obtain the following relations with the aid of Eqs. (29. a, b) :

$$
E_{m 0}^{(1)}=2(1-\nu) F_{m 0}^{(1)}, \quad E_{m 0}^{(2)}=-2(1-\nu) F_{m 0}^{(2)}
$$

Substituting the relations of Eqs. (29. a) to (30. b) into Eqs. (27.a c), the potential functions of displacement for the solution to the state of plane strain are obtained as:

$$
\begin{aligned}
& \phi_{0,0}=\sum_{m=2}^{\infty} D_{m 0}^{(1)} \cos m \theta r^{m}-\sum_{m=1}^{\infty} m F_{m 0}^{(1)} \cos m \theta\left\{\frac{r^{m+2}}{2}-(m+1) r^{m} z^{2}\right\}+\sum_{m=1}^{\infty} D_{m 0}^{(2)} \cos m \theta r^{-m} \\
& +\sum_{m=2}^{\infty} m F_{m 0}^{(2)} \cos m \theta\left\{\frac{r^{-m+2}}{2}+(m-1) r^{-m} z^{2}\right\} \\
& \phi_{1,0}=\sum_{m=1}^{\infty} F_{m 0}^{(1)} \cos m \theta\left\{\frac{r^{m+2}}{2}-(m+1) r^{m} z^{2}\right\}+\sum_{m=2}^{\infty} F_{m 0}^{(2)} \cos m \theta\left\{\frac{r^{-m+2}}{2}+(m-1) r^{-m} z^{2}\right\} \\
& \vartheta_{z, 0}=2(1-\nu) \sum_{m=1}^{\infty} F_{m 0}^{(1)} \sin m \theta\left\{\frac{r^{m+2}}{2}-(m+1) r^{m} z^{2}\right\}-2(1-\nu) \sum_{m=2}^{\infty} F_{m 0}^{(2)} \sin m \theta \\
& \cdot\left\{\frac{r^{-m+2}}{2}+(m-1) r^{-m} z^{2}\right\}
\end{aligned}
$$

The components of displacement obtained from Eqs. (31.a c) are as follows:

$$
\begin{aligned}
& 2 G u_{r}^{(0)}=\sum_{m=2}^{\infty} \cos m \theta\left\{m D_{m 0}^{(1)} r^{m-1}-F_{m 0}^{(2)}(m+2-4 \nu) r^{-m+1}\right\}-\sum_{m=1}^{\infty} \cos m \theta\left\{m D_{m 0}^{(2)} r^{-m-1}\right. \\
& \left.-F_{m 0}^{(1)}(m-2+4 \nu) r^{m+1}\right\} \text {. } \\
& 2 G u_{\theta}^{(0)}=-\sum_{m=2}^{\infty} \sin m \theta\left\{m D_{m 0}^{(1)} r^{m-1}+F_{m 0}^{(2)}(m-4+4 \nu) r^{-m+1}\right\}-\sum_{m=1}^{\infty} \sin m \theta\left\{m D_{m 0}^{(2)} r^{-m-1}\right. \\
& \left.+F_{m 0}^{(1)}(m+4-4 \nu) r^{m+1}\right\}
\end{aligned}
$$

Simple solutions to axi-symmetric problems are also needed for non-axially symmetric problems as additional solutions. They are

$$
\phi_{0,00}=D_{00} \log r, \phi_{1,00}=F_{00}\left(\frac{r^{2}}{2}-z^{2}\right), \phi_{3,00}=C_{00}\left(\frac{z^{2}}{2}-\frac{r^{2}}{4}\right)
$$

in which $D_{00}$ to $C_{00}$ are arbitrary constants. From the above equations, the components of displacement are

$$
2 G u_{r}^{(0,0)}=\frac{D_{00}}{r}-2(1-2 \nu) F_{00} r, 2 G u_{\theta}^{(0,0)}=0,2 G u_{z}^{(0,0)}=-2(1-2 \nu) C_{00} z
$$

Thus, the solution to the state of plane strain and the simple solution to axi-symmetric problems which is also needed for non-axially symmetric problems have been indicated as additional solutions.

\section{SOLUTIONS TO THREE-DIMENSIONAL ELASTICITY PROBLEMS IN SPHERICAL COORDINATES}

If we consider Eq. (4) in spherical coordinates $(\rho, \phi, \theta)$ as shown in Fig. 2, we have 


$$
2 G \boldsymbol{u}=\operatorname{grad}\left(\phi_{0}+\boldsymbol{r} \cdot \lambda\right)-4(1-\nu) \lambda+2 \operatorname{rot} \vartheta+\operatorname{grad} \chi
$$

in which

$$
\begin{aligned}
& \boldsymbol{u}=\left[u_{\rho}, u_{\phi}, u_{\theta}\right], \quad \boldsymbol{r}=[\rho, 0,0], \quad \lambda=\left[\lambda_{\rho}, \lambda_{\phi}, \lambda_{\theta}\right], \quad \vartheta=\left[\vartheta_{\rho}, \vartheta_{\phi}, \vartheta_{\theta}\right] \\
& \nabla^{2} \phi_{0}=-\frac{1-2 \nu}{1-\nu} \beta, \quad \nabla^{2} \chi=\frac{\alpha E}{1-\nu} T, \quad \nabla^{2} \lambda=0, \quad \nabla^{2} \vartheta=-\tau \\
& \nabla^{2} \equiv \frac{\partial^{2}}{\partial \rho^{2}}+\frac{2}{\rho} \frac{\partial}{\partial \rho}+\frac{1}{\rho^{2}} \frac{\partial^{2}}{\partial \phi^{2}}+\frac{\cot \phi}{\rho^{2}} \frac{\partial}{\partial \phi}+\frac{1}{\rho^{2} \sin ^{2} \phi} \frac{\partial^{2}}{\partial \theta^{2}}
\end{aligned}
$$

Laplace's equation (37.c) in the vector field can be expressed in the components of vector potential $\lambda$ as

$$
\begin{aligned}
& \nabla^{2} \lambda_{0}-\frac{2 \lambda_{0}}{\rho^{2}}-\frac{2 \cot \phi}{\rho^{2}} \lambda_{\phi}-\frac{2}{\rho^{2}} \frac{\partial \lambda_{\phi}}{\partial \phi}-\frac{2}{\rho^{2} \sin \phi} \frac{\partial \lambda_{\theta}}{\partial \theta}=0 . \\
& \nabla^{2} \lambda_{\phi}-\frac{\lambda_{\phi}}{\rho^{2} \sin ^{2} \phi}+\frac{2}{\rho^{2}} \frac{\partial \lambda_{0}}{\partial \phi}-\frac{2 \cot \phi}{\rho^{2} \sin \phi} \frac{\partial \lambda_{\theta}}{\partial \theta}=0 \ldots \ldots \ldots \ldots . . \\
& \nabla^{2} \lambda_{\theta}-\frac{\lambda_{\theta}}{\rho^{2} \sin ^{2} \phi}+\frac{2}{\rho^{2} \sin \phi} \frac{\partial \lambda_{\phi}}{\partial \theta}+\frac{2 \cot \phi}{\rho^{2} \sin \phi} \frac{\partial \lambda_{\phi}}{\partial \theta}=0 \ldots
\end{aligned}
$$

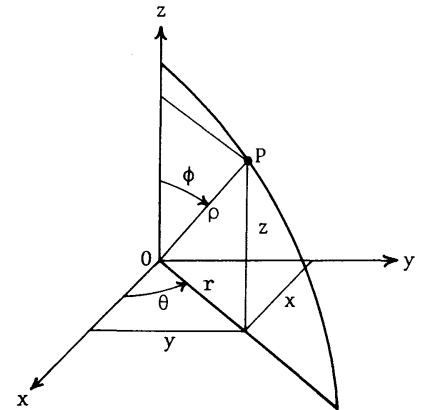

Fig. 2 Spherical coordinates.

From the above equations, we find a system of linear partial differential equations with three unknowns, that is, $\lambda_{\rho}, \lambda_{\phi}$ and $\lambda_{\theta}$. Though the system can be solved by a complicated modification with great length, the solutions must be checked by the investigation on an independent relation between each solution in the same manner as the solutions in the cylindrical coordinates. To avoid this difficulty, we put vector potential $\lambda$ in Eq. (36.c) into the following equation:

$$
\begin{aligned}
\lambda & =\boldsymbol{r}\left(\phi_{1}+2 \boldsymbol{r} \cdot \operatorname{grad} \phi_{1}\right)-(\boldsymbol{r} \cdot \boldsymbol{r}) \operatorname{grad} \phi_{1} \\
& =\left[\rho \frac{\partial\left(\rho \phi_{1}\right)}{\partial \rho},-\frac{\partial\left(\rho \phi_{1}\right)}{\partial \phi},-\frac{1}{\sin \phi} \frac{\partial\left(\rho \phi_{1}\right)}{\partial \theta}\right]
\end{aligned}
$$

in which

$$
\nabla^{2} \phi_{1}=0
$$

Relation (40) is exactly held to satisfy condition (37. c) that $\lambda$ must be the harmonic vector, as indicated in the following equation:

$$
\begin{aligned}
\nabla^{2} \lambda= & \nabla^{2}\left\{\boldsymbol{r}\left(\phi_{1}+2 \boldsymbol{r} \cdot \operatorname{grad} \phi_{1}\right)\right\}-\nabla^{2}\left\{(\boldsymbol{r} \cdot \boldsymbol{r}) \operatorname{grad} \phi_{1}\right\} \\
= & 2 \operatorname{grad}\left(\phi_{1}+2 \boldsymbol{r} \cdot \operatorname{grad} \phi_{1}\right)+\boldsymbol{r}\left\{5 \nabla^{2} \phi_{1}+2 \boldsymbol{r} \cdot \operatorname{grad} \nabla^{2} \phi_{1}\right\}-\operatorname{grad}\left\{2\left(\boldsymbol{r} \cdot \operatorname{grad} \phi_{1}\right)\right. \\
& \left.+(\boldsymbol{r} \cdot \boldsymbol{r}) \nabla^{2} \phi_{1}\right\}-2\left\{2 \operatorname{grad} \phi_{1}+(\boldsymbol{r} \cdot \operatorname{grad}) \operatorname{grad} \phi_{1}-\boldsymbol{r} \nabla^{2} \phi_{1}\right\} \\
= & 2\left\{3 \operatorname{grad} \phi_{1}+2(\boldsymbol{r} \cdot \operatorname{grad}) \operatorname{grad} \phi_{1}-3 \operatorname{grad} \phi_{1}-2(\boldsymbol{r} \cdot \operatorname{grad}) \operatorname{grad} \phi_{1}\right\} \\
& +\boldsymbol{r}\left\{5 \nabla^{2} \phi_{1}+2 \boldsymbol{r} \cdot \operatorname{grad} \nabla^{2} \phi_{1}\right\}-(\boldsymbol{r} \cdot \boldsymbol{r}) \operatorname{grad} \nabla^{2} \phi_{1} \\
= & \boldsymbol{r}\left\{5 \nabla^{2} \phi_{1}+2 \boldsymbol{r} \cdot \operatorname{grad} \nabla^{2} \phi_{1}\right\}-(\boldsymbol{r} \cdot \boldsymbol{r}) \operatorname{grad} \nabla^{2} \phi_{1}=\mathbf{0} \ldots \ldots \ldots \ldots \ldots \ldots \ldots \ldots \ldots \ldots \ldots \ldots \ldots \ldots \ldots \ldots \ldots \ldots \ldots \ldots
\end{aligned}
$$

In the derivation of the above equation, some formulae for vector calculus and the following relations are used :

$$
\begin{aligned}
& \nabla^{2}(\boldsymbol{r} \varphi)=\boldsymbol{r} \nabla^{2} \varphi+2 \operatorname{grad} \varphi, \operatorname{rot} \boldsymbol{r}=\mathbf{0}, \operatorname{div} \boldsymbol{r}=3,(\boldsymbol{A} \cdot \operatorname{grad}) \boldsymbol{r}=\boldsymbol{A} ; \\
& \nabla^{2}(\boldsymbol{r} \cdot \boldsymbol{A})=\boldsymbol{r} \cdot \nabla^{2} \boldsymbol{A}+2 \operatorname{div} \boldsymbol{A}
\end{aligned}
$$

in which $\varphi$ and $\boldsymbol{A}$ are arbitrary scalar and vector fields in the spherical coordinates, respectively. Substituting Eq. (40) into Eq. (35), we obtain

$$
\begin{aligned}
& 2 G \boldsymbol{u}=\operatorname{grad}\left\{\phi_{0}+\rho^{2} \frac{\partial\left(\rho \phi_{1}\right)}{\partial \rho}\right\}-4(1-\nu)\left[\rho \frac{\partial\left(\rho \phi_{1}\right)}{\partial \rho},-\frac{\partial\left(\rho \phi_{1}\right)}{\partial \phi},-\frac{1}{\sin \phi} \frac{\partial\left(\rho \phi_{1}\right)}{\partial \theta}\right] \\
& +2 \operatorname{rot} \vartheta+\operatorname{grad} \chi
\end{aligned}
$$

The components of displacement, from the above equation, are expressed as:

$$
2 G u_{\rho}=\frac{\partial \phi_{0}}{\partial \rho}+\rho \frac{\partial}{\partial \rho}\left\{\rho \frac{\partial\left(\rho \phi_{1}\right)}{\partial \rho}-(3-4 \nu)\left(\rho \phi_{1}\right)\right\}+\frac{2}{\rho \sin \phi}\left\{\frac{\partial\left(\vartheta_{\theta} \sin \phi\right)}{\partial \phi}-\frac{\partial \vartheta_{\phi}}{\partial \theta}\right\}+\frac{\partial \chi}{\partial \rho}
$$




$$
\begin{aligned}
& 2 G u_{\phi}=\frac{1}{\rho} \frac{\partial \phi_{0}}{\partial \phi}+\frac{\partial}{\partial \phi}\left\{\rho \frac{\partial\left(\rho \phi_{1}\right)}{\partial \rho}+4(1-\nu)\left(\rho \phi_{1}\right)\right\}+\frac{2}{\rho}\left\{\frac{1}{\sin \phi} \frac{\partial \vartheta_{\rho}}{\partial \theta}-\frac{\partial\left(\rho \vartheta_{\theta}\right)}{\partial \rho}\right\}+\frac{1}{\rho} \frac{\partial \chi}{\partial \phi} . \\
& 2 G u_{\theta}=\frac{1}{\rho \sin \phi} \frac{\partial \phi_{0}}{\partial \theta}+\frac{1}{\sin \phi} \frac{\partial}{\partial \theta}\left\{\rho \frac{\partial\left(\rho \phi_{1}\right)}{\partial \rho}+4(1-\nu)\left(\rho \phi_{1}\right)\right\}+\frac{2}{\rho}\left\{\frac{\partial\left(\rho \vartheta_{\phi}\right)}{\partial \rho}-\frac{\partial \vartheta_{o}}{\partial \phi}\right\} \\
& +\frac{1}{\rho \sin \phi} \frac{\partial \chi}{\partial \theta}
\end{aligned}
$$

in which the governing equations of $\phi_{0}, \chi, \vartheta$ and $\phi_{1}$ are expressed in Eqs. (37. a, b, d) and (41), respectively. In the case where $\boldsymbol{b}=\mathbf{0}$, on considering Eqs. (8. a, b) and independence from the potential function of displacement in Eq. (40), we can put that

$$
\vartheta_{\rho}=\rho \frac{\partial\left(\rho \phi_{2}\right)}{\partial \rho}, \vartheta_{\phi}=-\frac{\partial\left(\rho \phi_{2}\right)}{\partial \phi}, \vartheta_{\theta}=-\frac{1}{\sin \phi} \frac{\partial\left(\rho \phi_{2}\right)}{\partial \theta}
$$

Then, $\phi_{0}$ and $\phi_{2}$ must be harmonic functions as

$$
\nabla^{2} \phi_{0}=0, \quad \nabla^{2} \phi_{2}=0 \text {. }
$$

The components of displacement in case of zero body forces and zero temperature are obtained from putting $\chi=0$ and from substituting Eqs. $(46 . \mathrm{a} \sim \mathrm{c})$ into $\vartheta_{\rho}, \vartheta_{\phi}$ and $\vartheta_{\theta}$ in Eqs. $(45 . \mathrm{a} \sim \mathrm{c})$ as

$$
\begin{aligned}
& 2 G u_{\rho}=\frac{\partial \phi_{0}}{\partial \rho}+\rho \frac{\partial}{\partial \rho}\left\{\rho \frac{\partial\left(\rho \phi_{1}\right)}{\partial \rho}-(3-4 \nu)\left(\rho \phi_{1}\right)\right\} \\
& 2 G u_{\phi}=\frac{1}{\rho} \frac{\partial \phi_{0}}{\partial \phi}+\frac{\partial}{\partial \phi}\left\{\rho \frac{\partial\left(\rho \phi_{1}\right)}{\partial \rho}+4(1-\nu)\left(\rho \phi_{1}\right)\right\}+\frac{2}{\sin \phi} \frac{\partial}{\partial \theta}\left(3 \phi_{2}+2 \rho \frac{\partial \phi_{2}}{\partial \rho}\right) \\
& 2 G u_{\theta}=\frac{1}{\rho \sin \phi} \frac{\partial \phi_{0}}{\partial \theta}+\frac{1}{\sin \phi} \frac{\partial}{\partial \theta}\left\{\rho \frac{\partial\left(\rho \phi_{1}\right)}{\partial \rho}+4(1-\nu)\left(\rho \phi_{1}\right)\right\}-2 \frac{\partial}{\partial \phi}\left(3 \phi_{2}+2 \rho \frac{\partial \phi_{2}}{\partial \rho}\right)
\end{aligned}
$$

in which the governing equations of $\phi_{1}, \phi_{0}$ and $\phi_{2}$ are expressed in Eqs. (41) and (47. a, b), respectively. Hence, it is found that all potential functions of displacement reduce to the harmonic functions. This result yields simple and plain solutions to elasticity problems in the same manner as the solutions in the cylindrical coordinates. The harmonic functions in Eqs. (41) and (47.a, b) referring to the spherical coordinates are easily obtained. The potential functions of displacement for non-axially symmetric problems of hollow spheres are as follows:

$$
\begin{aligned}
& \phi_{0}=\sum_{n=0}^{\infty} \sum_{m=0}^{n} \cos m \theta P_{n}^{m}(\mu)\left(A_{n m} \rho^{n}+D_{n m} \rho^{-(n+1)}\right) \\
& \phi_{1}=\sum_{n=0}^{\infty} \sum_{m=0}^{n} \cos m \theta P_{n}^{m}(\mu)\left(E_{n m} \rho^{n}+C_{n m} \rho^{-(n+1)}\right) \\
& \phi_{2}=\sum_{n=0}^{\infty} \sum_{m=0}^{n} \sin m \theta P_{n}^{m}(\mu)\left(L_{n m} \rho^{n}+M_{n m} \rho^{-(n+1)}\right)
\end{aligned}
$$

in which $A_{n m}$ to $M_{n m}$ are arbitrary constants and

$$
\mu \equiv \cos \phi, \quad P_{n}^{m}(\mu)=\left(1-\mu^{2}\right)^{m / 2} \frac{d^{m} P_{n}(\mu)}{d \mu^{m}}
$$

The associated Legendre function of the second kind, that is, $Q_{n}^{m}(\mu)$ is eliminated from Eqs. (49. a c), because its function is not bounded at $\mu= \pm 1$. Substituting the potential functions of displacement in Eqs. $(49 . a \sim c)$ into Eqs. $(48 . a \sim c)$, the components of displacement excluding the rigid-body displacement are as follows:

$$
\begin{aligned}
& 2 G u_{\rho}=\sum_{n=2}^{\infty} \sum_{m=0}^{n} A_{n m} n \rho^{n-1} \cos m \theta P_{n}^{m}(\mu)+\sum_{n=1}^{\infty} \sum_{m=0}^{n} E_{n m}(n+1)(n-2+4 \nu) \rho^{n+1} \cos m \theta P_{n}^{m}(\mu) \\
& -2(1-2 \nu) E_{00} \rho-\sum_{n=1}^{\infty} \sum_{m=0}^{n} \cos m \theta P_{n}^{m}(\mu)\left\{D_{n m}(n+1) \rho^{-(n+2)}-C_{n m} n(n+3-4 \nu) \rho^{-n}\right\} \\
& -D_{00} \rho^{-2} \\
& 2 G u_{\phi}=-\sum_{n=2}^{\infty} \sum_{m=0}^{n} \cos m \theta\left\{A_{n m} \rho^{n-1} \sin \phi \frac{d P_{n}^{m}(\mu)}{d \mu}-2 L_{n m}(2 n+3) \rho^{n} \frac{m P_{n}^{m}(\mu)}{\sin \phi}\right\}
\end{aligned}
$$




$$
\begin{aligned}
& -\sum_{n=1}^{\infty} \sum_{m=0}^{n} E_{n m}(n+5-4 \nu) \rho^{n+1} \cos m \theta \sin \phi \frac{d P_{n}^{m}(\mu)}{d \mu}-\sum_{n=1}^{\infty} \sum_{m=0}^{n} \cos m \theta\left[\left\{D_{n m} \rho^{-(n+2)}\right.\right. \\
& \left.\left.-C_{n m}(n-4+4 \nu) \rho^{-n}\right\} \sin \phi \frac{d P_{n}^{m}(\mu)}{d \mu}+2 M_{n m}(2 n-1) \rho^{-(n+1)} \frac{m P_{n}^{m}(\mu)}{\sin \phi}\right] \text {. } \\
& 2 G u_{\theta}=-\sum_{n=2}^{\infty} \sum_{m=0}^{n} \sin m \theta\left\{A_{n m} \rho^{n-1} \frac{m P_{n}^{m}(\mu)}{\sin \phi}-2 L_{n m}(2 n+3) \rho^{n} \sin \phi \frac{d P_{n}^{m}(\mu)}{d \mu}\right\} \\
& -\sum_{n=1}^{\infty} \sum_{m=0}^{n} E_{n m}(n+5-4 \nu) \rho^{n+1} \sin m \theta \frac{m P_{n}^{m}(\mu)}{\sin \phi}-\sum_{n=1}^{\infty} \sum_{m=0}^{n} \sin m \theta\left[\left\{D_{n m} \rho^{-(n+2)}\right.\right. \\
& \left.\left.-C_{n m}(n-4+4 \nu) \rho^{-n}\right\} \frac{m P_{n}^{m}(\mu)}{\sin \phi}+2 M_{n m}(2 n-1) \rho^{-(n+1)} \sin \phi \frac{d P_{n}^{m}(\mu)}{d \mu}\right]
\end{aligned}
$$

in which the solutions with possitive powers and with negative powers to base $\rho$ are solutions to internal and to external problems, respectively. If we have components of strain in the spherical coordinates from the components of displacement in Eqs. (51. a c ), components of stress can be obtained from Hooke's law, though they are omitted here.

Thus, the generalized solution taking into account heat and body forces has been proposed as a solution to three-dimensional elasticity problems in the spherical coordinates. Also, the potential functions of displacement and the components of displacement for non-axially symmetric problems in the absence of a temperature and of body forces have been indicated as a particular case of its solution.

\section{CONCLUDING REMARKS}

As mentioned in Chaps. 3 and 5, Boussinesq's solution, Papkovich-Neuber's solution and Galerkin's solution heretofore in use have a defect that a determination of potential functions of displacement is complicated and an independent relation between each solution must be investigated, when their solutions are used in orthogonal curvilinear coordinates, for instance, cylindrical or spherical coordinates. Paying attention to it, this paper proposed simple and plain solutions to non-axially symmetric problems in both cylindrical and spherical coordinates and generalized Boussinesq's solution to taking into account heat and body forces. Relations (15) and (40) which replaced harmonic vector $\lambda$ with the vector field consisted of harmonic functions facilitated the determination of potential functions of displacement and needed no investigation on an independent relation between each solution. Therefore, we can say that these relations are available for constituting some solutions and are a new approach to three-dimensional elasticity problems. Also, the solutions in case of the absence of body forces have an advantage that all potential functions of displacement become harmonic functions like those in rectangular Cartesian coordinates. Furthermore, the solutions in Eqs. (22. a c ) in cylindrical coordinates have a wide range of application such as extends to the solution ${ }^{14)}$ to a transversely isotropic body. Though the complexity that rising and falling of the degree of Legendre polinomials according to internal and external problems is needed even for axi-symmetric problems is usually found, the solutions in Eqs. (48. a c) do not have this complexity even in case of non-axially symmetric problems by virtue of the replacements of Eqs. (40) and (46. a c ). The body force $b$, without its direct use, was expressed in the sum of the gradient of a scalar potential and the rotation of a vector potential with the aid of Helmholtz's theorem, because the replacements of $\lambda$ alone without coupling with $b$ were aimed throughout. Though some studies on elasticity problems of an anisotropic body have been recently reported, appropriate solutions to various types of an anisotropic body, needless to say, can not be found except for definite solutions to an isotropic body. In consideration of the premises, the present authors may conclude that the solutions proposed in this paper are fully available for three-dimensional elasticity problems.

\section{REFERENCES}

1) Miyamoto, H. : Three-Dimensional Problems in The Theory of Elasticity, p. 18, Syokabo, 1967 (in Japanese).

2) Mindlin, R. D. : Note on the Galerkin and Papkovitch stress functions, Bulletin of American Mathematical Society, Vol. XLII, 
pp. 373 376, 1936.

3) Nakahara, I. : Three-dimensional stress functions in the theory of elasticity, Proc. of 22nd Japan Nat. Cong. Appl. Mech., pp. 149 156, 1972.

4) Youngdahl, C. K. : On the completeness of a set of stress functions appropriate to the solution of elasticity problems in general cylindrical coordinates, Int. J. Engng. Sci., Vol. 7, pp. 61 79, 1969

5) Love, A.E.H. : A Treatise on the Mathematical Theory of Elasticity, 4th ed., pp. 276, Dover Publications, 1944.

6) Hasegawa, H. : Generalization of Love's solution to axi-symmetric elasticity problems, Trans. of JSME, Vol. 40, No. 337, pp. 2494 2504, 1974 (in Japanese).

7) Muki, R. : Three dimensional problem of elasticity for a semi-infinite solid with a tangential load on its surface, Trans. of JSME, Vol. 22, No. 119, pp. 468 474, 1956 (in Japanese).

8) Okumura, I. A. and Miyake, K. : On stresses and displacements in a thick circular ring sector plate based on the three-dimensional theory of elasticity, Theoretical and Applied Mechanics, Vol.29, pp. 127 138, 1981.

9) Okumura, I. A. and Miyake, K. : Stresses in a short hollow cylinder and a long hollow cylinder subjected to partial pressure on the side surface, Theoretical and Applied Mechanics, Vol. 30, pp. 81 93, 1981.

10) Lur'e, A. I. : Three-Dimensional Problems of the Theory of Elasticity, Chap. 6, Interscience Publishers, 1964.

11) Okumura, I. A. : On a method solving isothermal elastic problems and thermal stress problems of a hollow sphere, Proc. of JSCE, No. 307, pp. 1 15, 1981 (in Japanese).

12) Okumura, I. A. : A study on a stress analysis for non-axially symmetric, three-dimensional problems, unpublished dissertation for Dr. Eng., University of Hokkaido, pp. 38 43 and pp. 45 61, 1983 (in Japanese).

13) Iwahori, N. : Vector Analysis, pp. 222, Syokabo, 1975 (in Japanese).

14) Okumura, I. A. : On axi-symmetric deformation of transversely isotropic, finite circular cylinders, Proc. of 40 th Nat. Cong. of JSCE (First Division), pp. 37 38, 1985 (in Japanese). 